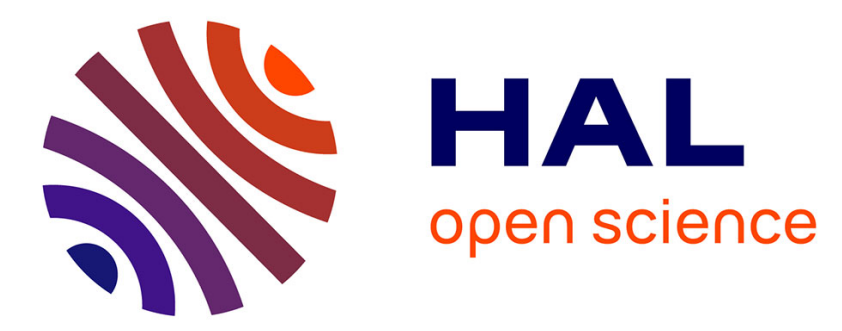

\title{
Global entropy weak solutions for general non-local traffic flow models with anisotropic kernel
}

\author{
Felisia Angela Chiarello, Paola Goatin
}

\section{To cite this version:}

Felisia Angela Chiarello, Paola Goatin. Global entropy weak solutions for general non-local traffic flow models with anisotropic kernel. ESAIM: Mathematical Modelling and Numerical Analysis, 2018, 52, pp.163-180. hal-01567575

\section{HAL Id: hal-01567575 \\ https://hal.inria.fr/hal-01567575}

Submitted on 24 Jul 2017

HAL is a multi-disciplinary open access archive for the deposit and dissemination of scientific research documents, whether they are published or not. The documents may come from teaching and research institutions in France or abroad, or from public or private research centers.
L'archive ouverte pluridisciplinaire $\mathbf{H A L}$, est destinée au dépôt et à la diffusion de documents scientifiques de niveau recherche, publiés ou non, émanant des établissements d'enseignement et de recherche français ou étrangers, des laboratoires publics ou privés. 


\title{
Global entropy weak solutions for general non-local traffic flow models with anisotropic kernel
}

\author{
Felisia Angela Chiarello* Paola Goatin*
}

July 24, 2017

\begin{abstract}
We prove the well-posedness of entropy weak solutions for a class of scalar conservation laws with non-local flux arising in traffic modeling. We approximate the problem by a Lax-Friedrichs scheme and we provide $L^{\infty}$ and BV estimates for the sequence of approximate solutions. Stability with respect to the initial data is obtained from the entropy condition through the doubling of variable technique. The limit model as the kernel support tends to infinity is also studied.
\end{abstract}

Key words: Scalar conservation laws, Anisotropic non-local flux, Lax-Friedrichs scheme, Traffic flow models.

\section{Introduction}

We consider the following scalar conservation law with non-local flux

$$
\partial_{t} \rho+\partial_{x}\left(f(\rho) v\left(J_{\gamma} * \rho\right)\right)=0, \quad x \in \mathbb{R}, t>0,
$$

where

$$
J_{\gamma} * \rho(t, x):=\int_{x}^{x+\gamma} J_{\gamma}(y-x) \rho(t, y) d y, \quad \gamma>0 .
$$

In (1.1), (1.2), we assume the following hypotheses:

$$
\begin{array}{ll}
f \in \mathbf{C}^{\mathbf{1}}\left(I ; \mathbb{R}^{+}\right), & I=[a, b] \subseteq \mathbb{R}^{+}, \\
v \in \mathbf{C}^{\mathbf{2}}\left(I ; \mathbb{R}^{+}\right) \text {s.t. } & v^{\prime} \leq 0, \\
J_{\gamma} \in \mathbf{C}^{\mathbf{1}}\left([0, \gamma] ; \mathbb{R}^{+}\right) \text {s.t. } & J_{\gamma}^{\prime} \leq 0 \text { and } \int_{0}^{\gamma} J_{\gamma}(x) d x:=J_{0}, \forall \gamma>0, \quad \lim _{\gamma \rightarrow \infty} J_{\gamma}(0)=0 .
\end{array}
$$

This class of equations includes in particular some vehicular traffic flow models $[4,10,15,18]$, where $\gamma>0$ is proportional to the look-ahead distance and the integral $J_{0}$ is the interaction strength (here assumed to be independent of $\gamma$ ). In this setting, the non-local dependence of the speed function $v$ can be interpreted as the reaction of drivers to a weighted mean of the downstream traffic density. Unlike similar non-local equations $[2,3,5,6,7,11,19]$, these models are characterized by the presence of an anisotropic discontinuous kernel, which makes general theoretical results $[1,2,3]$ inapplicable as such. On the other side, the specific monotonicity assumptions on the speed function $v$ and the kernel $J_{\gamma}$ ensure nice properties of the corresponding solutions, such as a strong maximum principle (both from below and above) and the absence of unphysical oscillations due to a sort of monotonicity preservation, which make the choice (1.2) interesting and justified from the modeling perspective.

Adding an initial condition

$$
\rho(0, x)=\rho_{0}(x), \quad x \in \mathbb{R},
$$

with $\rho_{0} \in \mathrm{BV}(\mathbb{R} ; I)$, entropy weak solutions of problem (1.1), (1.3), are intended the following sense $[2,3,13]$.

*Inria Sophia Antipolis - Méditerranée, Université Côte d'Azur, Inria, CNRS, LJAD, 2004 route des Lucioles - BP 93, 06902 Sophia Antipolis Cedex,France. E-mail: \{felisia.chiarello, paola.goatin\}@inria.fr 
Definition 1. A function $\rho \in\left(\mathbf{L}^{\mathbf{1}} \cap \mathbf{L}^{\infty} \cap B V\right)\left(\mathbb{R}^{+} \times \mathbb{R} ; I\right)$ is an entropy weak solution of (1.1), (1.3), if

$$
\begin{aligned}
\int_{0}^{+\infty} \int_{\mathbb{R}} & \left\{|\rho-\kappa| \varphi_{t}+\operatorname{sgn}(\rho-\kappa)(f(\rho)-f(\kappa)) v\left(J_{\gamma} * \rho\right) \varphi_{x}\right. \\
& \left.-\operatorname{sgn}(\rho-\kappa) f(\kappa) v^{\prime}\left(J_{\gamma} * \rho\right) \partial_{x}\left(J_{\gamma} * \rho\right) \varphi\right\} d x d t+\int_{\mathbb{R}}\left|\rho_{0}(x)-\kappa\right| \varphi(0, x) d x \geq 0
\end{aligned}
$$

for all $\varphi \in \mathbf{C}_{\mathbf{c}}^{\mathbf{1}}\left(\mathbb{R}^{2} ; \mathbb{R}^{+}\right)$and $\kappa \in \mathbb{R}$.

The main results of this paper are the following.

Theorem 1. Let hypotheses $(\mathbf{H})$ hold and $\rho_{0} \in B V(\mathbb{R} ; I)$. Then the Cauchy problem (1.1), (1.3), admits a unique weak entropy solution $\rho^{\gamma}$ in the sense of Definition 1, such that

$$
\min _{\mathbb{R}}\left\{\rho_{0}\right\} \leq \rho^{\gamma}(t, x) \leq \max _{\mathbb{R}}\left\{\rho_{0}\right\}, \quad \text { for a.e. } x \in \mathbb{R}, t>0 .
$$

Moreover, for any $T>0$ and $\tau>0$, the following estimates hold:

$$
\begin{aligned}
T V\left(\rho^{\gamma}(T, \cdot)\right) & \leq e^{C\left(J_{\gamma}\right) T} T V\left(\rho_{0}\right), \\
\left\|\rho^{\gamma}(T, \cdot)-\rho^{\gamma}(T-\tau, \cdot)\right\|_{\mathbf{L}^{1}} & \leq \tau e^{C\left(J_{\gamma}\right) T}\left(\left\|f^{\prime}\right\|\|v\|+J_{0}\|f\|\left\|v^{\prime}\right\|\right) T V\left(\rho_{0}\right),
\end{aligned}
$$

with $C\left(J_{\gamma}\right):=J_{\gamma}(0)\left(\left\|v^{\prime}\right\|\left(\left\|f^{\prime}\right\|\left\|\rho_{0}\right\|+2\|f\|\right)+\frac{7}{2} J_{0}\|f\|\left\|v^{\prime \prime}\right\|\right)$.

Above, and in the sequel, we use the compact notation $\|\cdot\|$ for $\|\cdot\|_{\mathbf{L}^{\infty}}$.

Corollary 2. Let hypotheses $(\mathbf{H})$ hold and $\rho_{0} \in B V(\mathbb{R} ; I)$. As $\gamma \rightarrow \infty$, the solution $\rho^{\gamma}$ of (1.1), (1.3) converges in the $\mathbf{L}_{\text {loc }}^{1}$-norm to the unique entropy weak solution of the classical Cauchy problem

$$
\begin{cases}\partial_{t} \rho+\partial_{x}(f(\rho) v(0))=0, & x \in \mathbb{R}, t>0 \\ \rho(0, x)=\rho_{0}(x), & x \in \mathbb{R} .\end{cases}
$$

In particular, we observe that $C\left(J_{\gamma}\right) \rightarrow 0$ in (1.6a) and (1.6b), allowing to recover the classical estimates.

The paper is organized as follows. Section 2 is devoted to the proof of the stability of solutions with respect to the initial data, based on a doubling of variable argument [13]. We observe that, for a close class of non-local equations, uniqueness of solutions has been recently derived in [12] relying on characteristics method and a fixed-point argument, thus avoiding the use of entropy conditions. In our setting, we prefer to keep the classical approach to pass to the limit $\gamma \rightarrow \infty$.

In Section 3 we derive existence of solutions through an approximation argument based on a Lax-Friedrichs type scheme. In particular, we prove accurate $\mathbf{L}^{\infty}$ and $\mathrm{BV}$ estimates on the approximate solutions, which allow to derive (1.5) and (1.6). We remark once again that these estimates heavily rely on the monotonicity properties of $J_{\gamma}$, and do not hold for general kernels, see $[2,4]$. Note that, regarding the Arrhenius look-ahead model [18], our result allows to establish a global well-posedness result and more accurate $\mathbf{L}^{\infty}$ estimates with respect to previous studies [15]. Moreover, to our knowledge, Corollary 2 provides the first convergence proof of a limiting procedure on the kernel support. We present some numerical tests illustrating this convergence in Section 4. Unfortunately, the limit $\gamma \rightarrow 0$, which was investigated numerically in $[2,4,10]$, remains unsolved, since in this case the constants in (1.6) blow up. 


\section{Uniqueness and stability of entropy solutions}

The Lipschitz continuous dependence of entropy solutions with respect to initial data can be derived using Kružkov's doubling of variable technique [13] as in [3, 4, 10].

Theorem 3. Under hypotheses $\mathbf{( H )}$, let $\rho, \sigma$ be two entropy solutions to (1.1) with initial data $\rho_{0}, \sigma_{0}$ respectively. Then, for any $T>0$ there holds

$$
\|\rho(t, \cdot)-\sigma(t, \cdot)\|_{\mathbf{L}^{1}} \leq e^{K T}\left\|\rho_{0}-\sigma_{0}\right\|_{\mathbf{L}^{1}} \quad \forall t \in[0, T],
$$

with $K$ given by $(2.5)$.

Proof. The functions $\rho$ and $\sigma$ are respectively entropy solutions of

$$
\begin{array}{lll}
\partial_{t} \rho(t, x)+\partial_{x}(f(\rho(t, x)) V(t, x))=0, & V:=v\left(\rho * J_{\gamma}\right), & \rho(0, x)=\rho_{0}(x), \\
\partial_{t} \sigma(t, x)+\partial_{x}(f(\sigma(t, x)) U(t, x))=0, & U:=v\left(\sigma * J_{\gamma}\right), & \sigma(0, x)=\sigma_{0}(x) .
\end{array}
$$

$V$ and $U$ are bounded measurable functions and are Lipschitz continuous w.r. to $x$, since $\rho, \sigma \in$ $\left(L^{1} \cap L^{\infty} \cap \mathrm{BV}\right)\left(\mathbb{R}^{+} \times \mathbb{R} ; \mathbb{R}\right)$. In particular, we have

$$
\left\|V_{x}\right\| \leq 2 J_{\gamma}(0)\left\|v^{\prime}\right\|\|\rho\|, \quad\left\|U_{x}\right\| \leq 2 J_{\gamma}(0)\left\|v^{\prime}\right\|\|\sigma\| .
$$

Using the classical doubling of variables technique introduced by Kruzkov, we obtain the following inequality:

$$
\begin{aligned}
\|\rho(T, \cdot)-\sigma(T, \cdot)\|_{\mathbf{L}^{1}} & \leq\left\|\rho_{0}-\sigma_{0}\right\|_{\mathbf{L}^{1}} \\
& +\left\|f^{\prime}\right\| \int_{0}^{T} \int_{\mathbb{R}}\left|\rho_{x}(t, x)\right||U(t, x)-V(t, x)| d x d t \\
& +\int_{0}^{T} \int_{\mathbb{R}}|f(\rho(t, x))|\left|U_{x}(t, x)-V_{x}(t, x)\right| d x d t .
\end{aligned}
$$

We observe that

$$
|U(t, x)-V(t, x)| \leq J_{\gamma}(0)\left\|v^{\prime}\right\|\|\rho(t, \cdot)-\sigma(t, \cdot)\|_{\mathbf{L}^{1}},
$$

and that for a.e. $x \in \mathbb{R}$

$$
\begin{aligned}
\left|U_{x}(t, x)-V_{x}(t, x)\right| \leq & \left(2\left(J_{\gamma}(0)\right)^{2}\left\|v^{\prime \prime}\right\|\|\rho(t, \cdot)\|+\left\|v^{\prime}\right\|\left\|J_{\gamma}^{\prime}\right\|\right)\|\rho(t, \cdot)-\sigma(t, \cdot)\|_{\mathbf{L}^{1}} \\
& +J_{\gamma}(0)\left\|v^{\prime}\right\|(|\rho-\sigma|(t, x+\gamma)+|\rho-\sigma|(t, x) .
\end{aligned}
$$

Plugging (2.3) and (2.4) into (2.2), we get

$$
\|\rho(T, \cdot)-\sigma(T, \cdot)\|_{\mathbf{L}^{1}} \leq\left\|\rho_{0}-\sigma_{0}\right\|_{\mathbf{L}^{1}}+K \int_{0}^{T}\|\rho(t, \cdot)-\sigma(t, \cdot)\|_{\mathbf{L}^{1}} d t
$$

with

$$
\begin{aligned}
K= & J_{\gamma}(0)\left\|v^{\prime}\right\|\left(\left\|f^{\prime}\right\| \sup _{t \in[0, T]}\|\rho(t, \cdot)\|_{\mathrm{BV}(\mathbb{R})}+2 \sup _{t \in[0, T]}\|f(\rho(t, \cdot))\|\right) \\
& +\sup _{t \in[0, T]}\|f(\rho(t, \cdot))\|_{1}\left(2\left(J_{\gamma}(0)\right)^{2}\left\|v^{\prime \prime}\right\| \sup _{t \in[0, T]}\|\rho(t, \cdot)\|+\left\|v^{\prime}\right\|\left\|J_{\gamma}^{\prime}\right\|\right) .
\end{aligned}
$$

By Gronwall's lemma, we get the thesis. 


\section{Existence}

\subsection{Lax-Friedrichs numerical scheme}

We discretize (1.1) on a fixed grid given by the cells interfaces $x_{j+\frac{1}{2}}=j \Delta x$ and the cells centers $x_{j}=(j-1 / 2) \Delta x$ for $j \in \mathbb{Z}$, taking a space step $\Delta x$ such that $\gamma=N \Delta x$ for some $N \in \mathbb{N}$, and $t^{n}=n \Delta t$ the time mesh. Our aim is to construct a finite volume approximate solution $\rho_{\Delta x}(t, x)=\rho_{j}^{n}$ for $(t, x) \in C_{j}^{n}=\left[t^{n}, t^{n+1}[\times] x_{j-1 / 2}, x_{j+1 / 2}\right]$. We approximate the initial datum $\rho_{0}$ with the piecewise constant function

$$
\rho_{j}^{0}=\frac{1}{\Delta x} \int_{x_{j-1 / 2}}^{x_{j+1 / 2}} \rho_{0}(x) d x .
$$

We denote $J_{\gamma}^{k}:=J_{\gamma}(k \Delta x)$ for $k=0, \ldots, N-1$ and set

$$
V_{j}^{n}:=v\left(c_{j}^{n}\right),
$$

where

$$
c_{j}^{n}:=\Delta x \sum_{k=0}^{N-1} J_{\gamma}^{k} \rho_{j+k}^{n} .
$$

The Lax-Friedrichs flux adapted to (1.1) is given by

$$
F_{j+1 / 2}^{n}:=\frac{1}{2} f\left(\rho_{j}^{n}\right) V_{j}^{n}+\frac{1}{2} f\left(\rho_{j+1}^{n}\right) V_{j+1}^{n}+\frac{\alpha}{2}\left(\rho_{j}^{n}-\rho_{j+1}^{n}\right),
$$

$\alpha \geq 0$ being the viscosity coefficient. In this way, we obtain the $N+2$ points finite volume scheme

$$
\rho_{j}^{n+1}=H\left(\rho_{j-1}^{n}, \ldots, \rho_{j+N}^{n}\right),
$$

where

$$
H\left(\rho_{j-1}, \ldots, \rho_{j+N}\right):=\rho_{j}+\frac{\lambda}{2} \alpha\left(\rho_{j-1}-2 \rho_{j}+\rho_{j+1}\right)+\frac{\lambda}{2}\left(f\left(\rho_{j-1}\right) V_{j-1}^{n}-f\left(\rho_{j+1}\right) V_{j+1}^{n}\right),
$$

with $\lambda=\Delta t / \Delta x$.

Assume $\rho_{i} \in I$ for $i=j-1, \ldots, j+N$, we can compute:

$$
\begin{aligned}
\frac{\partial H}{\partial \rho_{j-1}} & =\frac{\lambda}{2}\left(\alpha+V_{j-1} f^{\prime}\left(\rho_{j-1}\right)+\Delta x v^{\prime}\left(c_{j-1}\right) J_{\gamma}^{0} f\left(\rho_{j-1}\right)\right), \\
\frac{\partial H}{\partial \rho_{j}} & =1-\lambda\left(\alpha-\frac{1}{2} \Delta x f\left(\rho_{j-1}\right) v^{\prime}\left(c_{j-1}\right) J_{\gamma}^{1}\right) \geq 1-\lambda\left(\alpha+\frac{1}{2} \Delta x J_{\gamma}(0)\|f\|\left\|v^{\prime}\right\|\right), \\
\frac{\partial H}{\partial \rho_{j+1}} & =\frac{\lambda}{2}\left(\alpha+\Delta x f\left(\rho_{j-1}\right) v^{\prime}\left(c_{j-1}\right) J_{\gamma}^{2}-f^{\prime}\left(\rho_{j+1}\right) V_{j+1}-\Delta x f\left(\rho_{j+1}\right) v^{\prime}\left(c_{j+1}\right) J_{\gamma}^{0}\right), \\
\frac{\partial H}{\partial \rho_{j+k}} & =-\frac{\lambda}{2} \Delta x\left(f\left(\rho_{j+1}\right) v^{\prime}\left(c_{j+1}\right) J_{\gamma}^{k-1}-f\left(\rho_{j-1}\right) v^{\prime}\left(c_{j-1}\right) J_{\gamma}^{k+1}\right), \quad k=2, \ldots, N-2, \\
\frac{\partial H}{\partial \rho_{j+N-1}} & =-\frac{\lambda}{2} \Delta x f\left(\rho_{j+1}\right) v^{\prime}\left(c_{j+1}\right) J_{\gamma}^{N-2} \\
\frac{\partial H}{\partial \rho_{j+N}} & =-\frac{\lambda}{2} \Delta x f\left(\rho_{j+1}\right) v^{\prime}\left(c_{j+1}\right) J_{\gamma}^{N-1} .
\end{aligned}
$$


We have that (3.4e) and (3.4f) are non-negative. The positivity of (3.4b) follows assuming

$$
\Delta t \leq \frac{2}{2 \alpha+\Delta x J_{\gamma}(0)\|f\|\left\|v^{\prime}\right\|} \Delta x,
$$

which gives the CFL condition. Moreover, the bound

$$
\alpha \geq\left\|f^{\prime}\right\|\|v\|+\Delta x J_{\gamma}(0)\|f\|\left\|v^{\prime}\right\|
$$

guarantees the increasing monotonicity w.r.t. $\rho_{j-1}$ and $\rho_{j+1}$, respectively in (3.4a) and in (3.4c). The sign of $(3.4 \mathrm{~d})$ cannot be a priori determined and for this reason the numerical scheme (3.2), (3.3) is not monotone.

\subsection{Maximum principle and $L^{\infty}$ estimates}

Proposition 1. Let hypotheses $(\mathbf{H})$ hold. Given an initial datum $\rho_{j}^{0}, j \in \mathbb{Z}$, such that $\rho_{m}=$ $\min _{j \in \mathbb{Z}} \rho_{j}^{0} \in I$ and $\rho_{M}=\max _{j \in \mathbb{Z}} \rho_{j}^{0} \in I$, the finite volume approximation $\rho_{j}^{n}, j \in \mathbb{Z}$ and $n \in N$, constructed using the scheme (3.2), (3.3), satisfies the bounds

$$
\rho_{m} \leq \rho_{j}^{n} \leq \rho_{M},
$$

for all $j \in \mathbb{Z}$ and $n \in \mathbb{N}$, under the CFL condition (3.5).

Proof. We follow closely the idea in [4]. We start observing that

$$
\begin{aligned}
H\left(\rho_{m}, \rho_{m}, \rho_{m}, \rho_{j+2}, \ldots, \rho_{j+N-2}, \rho_{m}, \rho_{m}\right) & \geq \rho_{m}, \\
H\left(\rho_{M}, \rho_{M}, \rho_{M}, \rho_{j+2}, \ldots, \rho_{j+N-2}, \rho_{M}, \rho_{M}\right) & \leq \rho_{M} .
\end{aligned}
$$

Indeed, we get

$$
H\left(\rho_{m}, \rho_{m}, \rho_{m}, \rho_{j+2}, \ldots, \rho_{j+N-2}, \rho_{m}, \rho_{m}\right)=\rho_{m}+\frac{\lambda}{2} f\left(\rho_{m}\right)\left(V_{j-1}^{n}-V_{j+1}^{n}\right),
$$

and we have that

$$
V_{j-1}^{n}-V_{j+1}^{n}=v\left(c_{j-1}^{n}\right)-v\left(c_{j+1}^{n}\right)=-v^{\prime}(\xi) \Delta x \sum_{k=0}^{N-1} J_{\gamma}^{k}\left(\rho_{j+k+1}-\rho_{j+k-1}\right) \geq 0,
$$

for some $\xi$ is between $c_{j-1}^{n}$ and $c_{j+1}^{n}$. Indeed, due to the non-increasing monotonicity of $J_{\gamma}$, we observe that

$$
\begin{aligned}
\sum_{k=0}^{N-1} J_{\gamma}^{k}\left(\rho_{j+k+1}-\rho_{j+k-1}\right) & =\rho_{m}\left(J_{\gamma}^{N-2}+J_{\gamma}^{N-1}-J_{\gamma}^{0}-J_{\gamma}^{1}\right)+\sum_{k=1}^{N-2} \rho_{j+k}\left(J_{\gamma}^{k-1}-J_{\gamma}^{k+1}\right) \\
& \geq \rho_{m} \sum_{k=1}^{N-2}\left(J_{\gamma}^{k-1}-J_{\gamma}^{k+1}\right) \geq 0
\end{aligned}
$$

In this way we have the inequality (3.7) and the same procedure leads to (3.8).

Consider now the points

$$
R_{j}^{n}=\left(\rho_{j-1}^{n}, \ldots, \rho_{j+N}^{n}\right)
$$


and

$$
R_{m}^{n}=\left(\rho_{m}, \rho_{m}, \rho_{m}, \rho_{j+2}^{n}, \ldots, \rho_{j+N-2}^{n}, \rho_{m}, \rho_{m}\right) .
$$

Applying the mean value theorem and using (3.7) one has

$$
\begin{array}{r}
\rho_{j}^{n+1}=H\left(R_{j}^{n}\right)=H\left(R_{m}^{n}\right)+\nabla H\left(R_{\xi}\right) \cdot\left(R_{j}^{n}-R_{m}^{n}\right) \\
\geq \rho_{m}+\nabla H\left(R_{\xi}\right) \cdot\left(R_{j}^{n}-R_{m}^{n}\right),
\end{array}
$$

for $R_{\xi}=(1-\xi) R_{m}^{n}+\xi R_{j}^{n}$, for some $\xi \in[0,1]$. We note that

$$
\frac{\partial H}{\partial \rho_{j+k}}\left(R_{\xi}\right)\left(R_{j}^{n}-R_{m}^{n}\right)_{k}=0, \quad k=2, \ldots, N-2,
$$

since $\left(R_{j}^{n}-R_{m}^{n}\right)_{k}=0$ for $k=2, \ldots, N-2$. Assuming (3.5) and (3.6), we conclude

$$
\nabla H\left(R_{\xi}\right) \cdot\left(R_{j}^{n}-R_{m}^{n}\right) \geq 0
$$

which by (3.9) implies that $\rho_{j}^{n+1} \geq \rho_{m}$.

Similarly we can prove the upper bound by considering

$$
R_{M}^{n}=\left(\rho_{M}, \rho_{M}, \rho_{M}, \rho_{j+2}^{n}, \ldots, \rho_{j+N-2}^{n}, \rho_{M}, \rho_{M}\right)
$$

and (3.8).

\subsection{BV estimates}

The approximate solutions constructed using adapted Lax-Friedrichs numerical scheme have uniformly bounded total variation.

Proposition 2. Let hypotheses $(\mathbf{H})$ hold, $\rho_{0} \in B V(\mathbb{R} ; I)$, and let $\rho_{\Delta x}$ be constructed using (3.2), (3.3). If

$$
\begin{aligned}
\alpha & \geq\left\|f^{\prime}\right\|\|v\|+\Delta x J_{\gamma}(0)\left\|v^{\prime}\right\|\left(\|f\|+\left\|f^{\prime}\right\|\left\|\rho_{0}\right\|\right), \\
\Delta t & \leq \frac{2 \Delta x}{2 \alpha+\Delta x J_{\gamma}(0)\left\|v^{\prime}\right\|\left(\|f\|+\left\|f^{\prime}\right\|\left\|\rho_{0}\right\|\right)},
\end{aligned}
$$

then for every $T>0$ the following discrete space $B V$ estimate holds

$$
T V\left(\rho_{\Delta x}\right)(T, \cdot) \leq e^{C\left(J_{\gamma}\right) T} T V\left(\rho_{0}\right),
$$

where $C\left(J_{\gamma}\right):=J_{\gamma}(0)\left(\left\|v^{\prime}\right\|\left(\left\|f^{\prime}\right\|\left\|\rho_{0}\right\|+2\|f\|\right)+\frac{7}{2} J_{0}\|f\|\left\|v^{\prime \prime}\right\|\right)$.

Proof. At the mesh cell $C_{j}^{n}$ there holds

$$
\rho_{j}^{n+1}=\rho_{j}+\frac{\lambda \alpha}{2}\left(\rho_{j-1}-2 \rho_{j}+\rho_{j+1}\right)+\frac{\lambda}{2}\left(f\left(\rho_{j-1}\right) V_{j-1}-f\left(\rho_{j+1}\right) V_{j+1}\right),
$$

and at $C_{j+1}^{n}$

$$
\rho_{j+1}^{n+1}=\rho_{j+1}+\frac{\lambda \alpha}{2}\left(\rho_{j}-2 \rho_{j+1}+\rho_{j+2}\right)+\frac{\lambda}{2}\left(f\left(\rho_{j}\right) V_{j}-f\left(\rho_{j+2}\right) V_{j+2}\right),
$$


where we omitted the index $n$ to simplify the notation. Computing the difference between $\rho_{j+1}^{n+1}$ and $\rho_{j}^{n+1}$ and setting $\Delta_{j+k-1 / 2}^{n}=\rho_{j+k}^{n}-\rho_{j+k-1}^{n}$ for $k=0, \ldots, N+1$ we get:

$$
\begin{aligned}
& \Delta_{j+1 / 2}^{n+1}=\Delta_{j+1 / 2}+\frac{\lambda \alpha}{2}\left[\Delta_{j-1 / 2}-2 \Delta_{j+1 / 2}+\Delta_{j+3 / 2}\right] \\
& +\frac{\lambda}{2}\left[f\left(\rho_{j}\right) V_{j} \pm f\left(\rho_{j-1}\right) V_{j}-f\left(\rho_{j-1}\right) V_{j-1}-f\left(\rho_{j+2}\right) V_{j+2} \pm f\left(\rho_{j+1}\right) V_{j+2}+f\left(\rho_{j+1}\right) V_{j+1}\right]
\end{aligned}
$$

Applying the mean value theorem we can rewrite (3.11) as:

$$
\begin{aligned}
\Delta_{j+1 / 2}^{n+1}= & \Delta_{j+1 / 2}+\frac{\lambda \alpha}{2}\left[\Delta_{j-1 / 2}-2 \Delta_{j+1 / 2}+\Delta_{j+3 / 2}\right] \\
& +\frac{\lambda}{2}\left[V_{j} f^{\prime}\left(\zeta_{j-1 / 2}\right) \Delta_{j-1 / 2}+f\left(\rho_{j-1}\right)\left(V_{j}-V_{j-1}\right)\right. \\
& \left.-V_{j+2} f^{\prime}\left(\zeta_{j+3 / 2}\right) \Delta_{j+3 / 2}+f\left(\rho_{j+1}\right)\left(V_{j+1}-V_{j+2}\right)\right] .
\end{aligned}
$$

where $\zeta_{j-1 / 2}$ is between $\rho_{j-1}$ and $\rho_{j}$. Applying the mean value theorem we have

$$
\begin{gathered}
V_{j}-V_{j-1}=v^{\prime}\left(\xi_{j-1 / 2}\right) \Delta x \sum_{k=0}^{N-1} J_{\gamma}^{k} \Delta_{j+k-\frac{1}{2}}, \\
V_{j+2}-V_{j+1}=v^{\prime}\left(\xi_{j+3 / 2}\right) \Delta x \sum_{k=0}^{N-1} J_{\gamma}^{k} \Delta_{j+k+\frac{3}{2}},
\end{gathered}
$$

where $\xi_{j+3 / 2}$ is between $\sum_{k=0}^{N-1} J_{\gamma}^{k} \rho_{j+k+1}$ and $\sum_{k=0}^{N-1} J_{\gamma}^{k} \rho_{j+k+2}$. In this way we obtain

$$
\begin{aligned}
\Delta_{j+1 / 2}^{n+1}= & \frac{\lambda}{2}\left[\alpha+V_{j} f^{\prime}\left(\zeta_{j-1 / 2}\right)+\Delta x J_{\gamma}^{0} v^{\prime}\left(\xi_{j-1 / 2}\right) f\left(\rho_{j-1}\right)\right] \Delta_{j-1 / 2} \\
+ & {\left[1-\lambda \alpha+\frac{\lambda}{2} \Delta x J_{\gamma}^{1} v^{\prime}\left(\xi_{j-1 / 2}\right) f\left(\rho_{j-1}\right)\right] \Delta_{j+1 / 2} } \\
+ & \frac{\lambda}{2}\left[\alpha-V_{j+2} f^{\prime}\left(\zeta_{j+3 / 2}\right)-\Delta x J_{\gamma}^{0} f\left(\rho_{j+1}\right) v^{\prime}\left(\xi_{j+3 / 2}\right)\right. \\
& \left.\quad+\Delta x J_{\gamma}^{2} v^{\prime}\left(\xi_{j-1 / 2}\right) f\left(\rho_{j-1}\right)\right] \Delta_{j+3 / 2} \\
+ & \frac{\lambda}{2} \Delta x f\left(\rho_{j-1}\right) v^{\prime}\left(\xi_{j-1 / 2}\right) \sum_{k=3}^{N-1} J_{\gamma}^{k} \Delta_{j+k-1 / 2} \\
& -\frac{\lambda}{2} \Delta x f\left(\rho_{j+1}\right) v^{\prime}\left(\xi_{j+3 / 2}\right) \sum_{k=1}^{N-1} J_{\gamma}^{k} \Delta_{j+k+3 / 2} .
\end{aligned}
$$

Rearranging the indexes in (3.13d) and (3.13e) we obtain

$$
\begin{aligned}
(3.13 \mathrm{~d})+(3.13 \mathrm{e})= & \frac{\lambda}{2} \Delta x \sum_{k=2}^{N-2}\left[f\left(\rho_{j-1}\right) v^{\prime}\left(\xi_{j-1 / 2}\right) J_{\gamma}^{k+1}-f\left(\rho_{j+1}\right) v^{\prime}\left(\xi_{j+3 / 2}\right) J_{\gamma}^{k-1}\right] \Delta_{j+k+1 / 2} \\
& -\frac{\lambda}{2} \Delta x f\left(\rho_{j+1}\right) v^{\prime}\left(\xi_{j+3 / 2}\right) J_{\gamma}^{N-2} \Delta_{j+N-1 / 2} \\
& -\frac{\lambda}{2} \Delta x f\left(\rho_{j+1}\right) v^{\prime}\left(\xi_{j+3 / 2}\right) J_{\gamma}^{N-1} \Delta_{j+N+1 / 2}
\end{aligned}
$$


Noting that adding and subtracting $f\left(\rho_{j-1}\right) J_{\gamma}^{k-1} v^{\prime}\left(\xi_{j-1 / 2}\right)$ in the sum we have

$$
\begin{aligned}
& f\left(\rho_{j-1}\right) v^{\prime}\left(\xi_{j-1 / 2}\right) J_{\gamma}^{k+1}-f\left(\rho_{j+1}\right) v^{\prime}\left(\xi_{j+3 / 2}\right) J_{\gamma}^{k-1} \\
= & f\left(\rho_{j-1}\right) v^{\prime}\left(\xi_{j-1 / 2}\right)\left(J_{\gamma}^{k+1}-J_{\gamma}^{k-1}\right) \\
& +J_{\gamma}^{k-1}\left(f\left(\rho_{j-1}\right) v^{\prime}\left(\xi_{j-1 / 2}\right) \pm f\left(\rho_{j-1}\right) v^{\prime}\left(\xi_{j+3 / 2}\right)-f\left(\rho_{j+1}\right) v^{\prime}\left(\xi_{j+3 / 2}\right)\right) \\
= & f\left(\rho_{j-1}\right) v^{\prime}\left(\xi_{j-1 / 2}\right)\left(J_{\gamma}^{k+1}-J_{\gamma}^{k-1}\right)+J_{\gamma}^{k-1} f\left(\rho_{j-1}\right)\left(v^{\prime}\left(\xi_{j-1 / 2}\right)-v^{\prime}\left(\xi_{j+3 / 2}\right)\right) \\
& -J_{\gamma}^{k-1} v^{\prime}\left(\xi_{j+3 / 2}\right) f^{\prime}\left(\zeta_{j}\right)\left(\Delta_{j-1 / 2}+\Delta_{j+1 / 2}\right),
\end{aligned}
$$

with $\zeta_{j}$ is between $\rho_{j-1}$ and $\rho_{j+1}$. Therefore we get

$$
\begin{aligned}
\Delta_{j+1 / 2}^{n+1}= & \frac{\lambda}{2}\left[\alpha+V_{j} f^{\prime}\left(\zeta_{j-1 / 2}\right)+\Delta x J_{\gamma}^{0} v^{\prime}\left(\xi_{j-1 / 2}\right) f\left(\rho_{j-1}\right)\right. \\
& \left.-\Delta x v^{\prime}\left(\xi_{j+3 / 2}\right) f^{\prime}\left(\zeta_{j}\right) \sum_{k=2}^{N-2} J_{\gamma}^{k-1} \Delta_{j+k+1 / 2}\right] \Delta_{j-1 / 2} \\
& +\left[1-\lambda \alpha+\frac{\lambda}{2} \Delta x J_{\gamma}^{1} v^{\prime}\left(\xi_{j-1 / 2}\right) f\left(\rho_{j-1}\right)\right. \\
& \left.\quad-\frac{\lambda}{2} \Delta x v^{\prime}\left(\xi_{j+3 / 2}\right) f^{\prime}\left(\zeta_{j}\right) \sum_{k=2}^{N-2} J_{\gamma}^{k-1} \Delta_{j+k+1 / 2}\right] \Delta_{j+1 / 2} \\
& +\frac{\lambda}{2}\left[\alpha-V_{j+2} f^{\prime}\left(\zeta_{j+3 / 2}\right)-\Delta x J_{\gamma}^{0} f\left(\rho_{j+1}\right) v^{\prime}\left(\xi_{j+3 / 2}\right)\right. \\
& \left.+\Delta x J_{\gamma}^{2} f\left(\rho_{j-1}\right) v^{\prime}\left(\xi_{j-1 / 2}\right)\right] \Delta_{j+3 / 2} \\
& +\frac{\lambda}{2} \Delta x \sum_{k=2}^{N-2}\left[f\left(\rho_{j-1}\right) v^{\prime}\left(\xi_{j-1 / 2}\right)\left(J_{\gamma}^{k+1}-J_{\gamma}^{k-1}\right)\right. \\
& \left.+J_{\gamma}^{k-1} f\left(\rho_{j-1}\right)\left(v^{\prime}\left(\xi_{j+1 / 2}\right)-v^{\prime}\left(\xi_{j+3 / 2}\right)\right)\right] \Delta_{j+k+1 / 2} \\
& -\frac{\lambda}{2} \Delta x f\left(\rho_{j+1}\right) v^{\prime}\left(\xi_{j+3 / 2}\right) J_{\gamma}^{N-2} \Delta_{j+N-1 / 2} \\
& -\frac{\lambda}{2} \Delta x f\left(\rho_{j+1}\right) v^{\prime}\left(\xi_{j+3 / 2}\right) J_{\gamma}^{N-1} \Delta_{j+N+1 / 2} .
\end{aligned}
$$

Observe that the assumption $\alpha \geq\left\|f^{\prime}\right\|\|v\|+\Delta x J_{\gamma}(0)\left\|v^{\prime}\right\|\left(\|f\|+\left\|f^{\prime}\right\|\left\|\rho_{0}\right\|\right)$ guarantees the positivity of (3.14a). Similarly for (3.14c) we get $\alpha \geq\left\|f^{\prime}\right\|\|v\|+\Delta x J_{\gamma}(0)\|f\|\left\|v^{\prime}\right\|$ and for (3.14b) we have the following CFL condition

$$
\Delta t \leq \frac{2 \Delta x}{2 \alpha+\Delta x J_{\gamma}(0)\left\|v^{\prime}\right\|\left(\|f\|+\left\|f^{\prime}\right\|\left\|\rho_{0}\right\|\right)} .
$$

Rearranging the indexes and taking the absolute values

$$
\begin{aligned}
& \sum_{j}\left|\Delta_{j+1 / 2}^{n+1}\right| \\
\leq & \sum_{j}\left|\Delta_{j+1 / 2}\right|
\end{aligned}
$$




$$
\begin{aligned}
& \times\left[\frac{\lambda}{2}\left(\alpha+V_{j+1} f^{\prime}\left(\zeta_{j+1 / 2}\right)+\Delta x J_{\gamma}^{0} v^{\prime}\left(\xi_{j+1 / 2}\right) f\left(\rho_{j}\right)-\Delta x v^{\prime}\left(\xi_{j+5 / 2}\right) f^{\prime}\left(\zeta_{j+1}\right) \sum_{k=2}^{N-2} J_{\gamma}^{k-1} \Delta_{j+k+3 / 2}\right)\right. \\
& +1-\lambda \alpha+\frac{\lambda}{2} \Delta x J_{\gamma}^{1} v^{\prime}\left(\xi_{j-1 / 2}\right) f\left(\rho_{j-1}\right)-\frac{\lambda}{2} \Delta x v^{\prime}\left(\xi_{j+3 / 2}\right) f^{\prime}\left(\zeta_{j}\right) \sum_{k=2}^{N-2} J_{\gamma}^{k-1} \Delta_{j+k+1 / 2} \\
& +\frac{\lambda}{2}\left(\alpha-V_{j+1} f^{\prime}\left(\zeta_{j+1 / 2}\right)-\Delta x J_{\gamma}^{0} f\left(\rho_{j}\right) v^{\prime}\left(\xi_{j+1 / 2}\right)+\Delta x J_{\gamma}^{2} v^{\prime}\left(\xi_{j-3 / 2}\right) f\left(\rho_{j-2}\right)\right) \\
& +\frac{\lambda}{2} \Delta x\left(\sum_{k=2}^{N-2} f\left(\rho_{j-k-1}\right) v^{\prime}\left(\xi_{j-k-1 / 2}\right)\left(J_{\gamma}^{k+1}-J_{\gamma}^{k-1}\right)+J_{\gamma}^{k-1} f\left(\rho_{j-k-1}\right)\left|v^{\prime}\left(\xi_{j-k-1 / 2}\right)-v^{\prime}\left(\xi_{j-k+3 / 2}\right)\right|\right) \\
& \left.-\frac{\lambda}{2} \Delta x f\left(\rho_{j-N+2}\right) v^{\prime}\left(\xi_{j-N+5 / 2}\right) J_{\gamma}^{N-2}-\frac{\lambda}{2} \Delta x f\left(\rho_{j-N+1}\right) v^{\prime}\left(\xi_{j-N+3 / 2}\right) J_{\gamma}^{N-1}\right]
\end{aligned}
$$

Due to some cancellations, the coefficient of the right-hand side of (3.16) becomes

$$
\begin{aligned}
1+\frac{\Delta t}{2}[ & -v^{\prime}\left(\xi_{j+5 / 2}\right) f^{\prime}\left(\zeta_{j+1}\right) \sum_{k=2}^{N-2} J_{\gamma}^{k-1} \Delta_{j+k+3 / 2}-v^{\prime}\left(\xi_{j+3 / 2}\right) f^{\prime}\left(\zeta_{j}\right) \sum_{k=2}^{N-2} J_{\gamma}^{k-1} \Delta_{j+k+1 / 2} \\
& +J_{\gamma}^{1} v^{\prime}\left(\xi_{j-1 / 2}\right) f\left(\rho_{j-1}\right)+J_{\gamma}^{2} v^{\prime}\left(\xi_{j-3 / 2}\right) f\left(\rho_{j-2}\right) \\
& +\left(\sum_{k=2}^{N-2} f\left(\rho_{j-k-1}\right) v^{\prime}\left(\xi_{j-k-1 / 2}\right)\left(J_{\gamma}^{k+1}-J_{\gamma}^{k-1}\right)+J_{\gamma}^{k-1} f\left(\rho_{j-k-1}\right)\left|v^{\prime}\left(\xi_{j-k-1 / 2}\right)-v^{\prime}\left(\xi_{j-k+3 / 2}\right)\right|\right) \\
& \left.-f\left(\rho_{j-N+2}\right) v^{\prime}\left(\xi_{j-N+5 / 2}\right) J_{\gamma}^{N-2}-f\left(\rho_{j-N+1}\right) v^{\prime}\left(\xi_{j-N+3 / 2}\right) J_{\gamma}^{N-1}\right] .
\end{aligned}
$$

Following [9, pp. 11-12], applying the mean value theorem to $v^{\prime}$ and using the monotonicity of the kernel $J_{\gamma}$, we have

$$
\left|v^{\prime}\left(\xi_{j-k-1 / 2}\right)-v^{\prime}\left(\xi_{j-k+3 / 2}\right)\right| \leq 7 J_{\gamma}(0)\left\|v^{\prime \prime}\right\| \Delta x
$$

Therefore we have

$$
\begin{aligned}
(3.17) \leq 1+\frac{\Delta t}{2}[ & 2 J_{\gamma}(0)\left\|v^{\prime}\right\|\left\|f^{\prime}\right\|\left\|\rho_{0}\right\|+2 J_{\gamma}(0)\left\|v^{\prime}\right\|\|f\| \\
& +\left\|v^{\prime}\right\|\|f\| \underbrace{\sum_{k=2}^{N-2}\left(J_{\gamma}^{k-1}-J_{\gamma}^{k+1}\right)}_{\sum_{k=1}^{N-3} J^{k}-\sum_{k=3}^{N-1} J_{\gamma}^{k}}+7 J_{\gamma}(0)\left\|v^{\prime \prime}\right\|\|f\| \underbrace{\Delta x \sum_{k=2}^{N-2} J_{\gamma}^{k-1}}_{\leq J_{0}}] .
\end{aligned}
$$

Substituting in (3.16) we get

$$
\sum_{j}\left|\Delta_{j+1 / 2}^{n+1}\right| \leq\left[1+\frac{\Delta t}{2}\left(2 J_{\gamma}(0)\left\|v^{\prime}\right\|\left(\left\|f^{\prime}\right\|\left\|\rho_{0}\right\|+2\|f\|\right)+7 J_{\gamma}(0) J_{0}\|f\|\left\|v^{\prime \prime}\right\|\right)\right] \sum_{j}\left|\Delta_{j+1 / 2}^{n}\right|,
$$

therefore we recover the following estimate for the total variation

$$
\operatorname{TV}\left(\rho_{\Delta x}(T, \cdot)\right) \leq\left[1+\frac{\Delta t}{2}\left(2 J_{\gamma}(0)\left\|v^{\prime}\right\|\left(\left\|f^{\prime}\right\|\left\|\rho_{0}\right\|+2\|f\|\right)+7 J_{\gamma}(0) J_{0}\|f\|\left\|v^{\prime \prime}\right\|\right)\right]^{T / \Delta t} \operatorname{TV}\left(\rho_{\Delta x}(0, \cdot)\right)
$$




$$
\leq e^{J_{\gamma}(0)\left(\left\|v^{\prime}\right\|\left(\left\|f^{\prime}\right\|\left\|\rho_{0}\right\|+2\|f\|\right)+\frac{7}{2} J_{0}\|f\|\left\|v^{\prime \prime}\right\|\right) T} \operatorname{TV}\left(\rho_{0}\right)
$$

From Proposition 2, the following space-time BVestimate can be derived (see [8, Corollary 5.1]).

Corollary 4. Let hypotheses $(\mathbf{H})$ hold, $\rho_{0} \in B V(\mathbb{R} ; I)$, and $\rho_{\Delta x}$ be given by (3.2), (3.3). If

$$
\begin{aligned}
\alpha & \geq\left\|f^{\prime}\right\|\|v\|+\Delta x J_{\gamma}(0)\left\|v^{\prime}\right\|\left(\|f\|+\left\|f^{\prime}\right\|\left\|\rho_{0}\right\|\right), \\
\Delta t & \leq \frac{2 \Delta x}{2 \alpha+\Delta x J_{\gamma}(0)\left\|v^{\prime}\right\|\left(\|f\|+\left\|f^{\prime}\right\|\left\|\rho_{0}\right\|\right)},
\end{aligned}
$$

then, for every $T>0, \rho_{\Delta x}$ satisfies the following Total Variation estimate in space and time

$$
\begin{aligned}
& T V\left(\rho_{\Delta x} ; \mathbb{R} \times[0, T]\right) \\
\leq & T e^{C\left(J_{\gamma}\right) T}\left(1+\left\|f^{\prime}\right\|\|v\|+\frac{1}{2} \Delta x J_{\gamma}(0)\left\|v^{\prime}\right\|\left(5\|f\|+\left\|f^{\prime}\right\|\left\|\rho_{0}\right\|\right)+J_{0}\|f\|\left\|v^{\prime}\right\|\right) T V\left(\rho_{0}\right) .
\end{aligned}
$$

Proof. Let us fix $T \in \mathbb{R}^{+}$. If $T \leq \Delta t$, then $\operatorname{TV}\left(\rho_{\Delta x} ;[0, T] \times \mathbb{R}\right) \leq T \mathrm{TV}\left(\rho_{0}\right)$. Let us assume now that $T>\Delta t$. Let $M \in \mathbb{N} \backslash\{0\}$ such that $M \Delta t<T \leq(M+1) \Delta t$. Then

$\operatorname{TV}\left(\rho_{\Delta x} ; \mathbb{R} \times[0, T]\right)=\sum_{n=0}^{M-1} \sum_{j \in \mathbb{Z}} \Delta t\left|\rho_{j+1}^{n}-\rho_{j}^{n}\right|+(T-M \Delta t) \sum_{j \in \mathbb{Z}}\left|\rho_{j+1}^{M}-\rho_{j}^{M}\right|+\sum_{n=0}^{M-1} \sum_{j \in \mathbb{Z}} \Delta x\left|\rho_{j}^{n+1}-\rho_{j}^{n}\right|$.

The spatial BV estimate yields

$$
\sum_{n=0}^{M-1} \sum_{j \in \mathbb{Z}} \Delta t\left|\rho_{j+1}^{n}-\rho_{j}^{n}\right|+(T-M \Delta t) \sum_{j \in \mathbb{Z}}\left|\rho_{j+1}^{M}-\rho_{j}^{M}\right| \leq T e^{C\left(J_{\gamma}\right) T} \operatorname{TV}\left(\rho_{0}\right)
$$

where $C\left(J_{\gamma}\right)$ is the constant in Proposition 2. We are left to bound the term

$$
\sum_{n=0}^{M-1} \sum_{j \in \mathbb{Z}} \Delta x\left|\rho_{j}^{n+1}-\rho_{j}^{n}\right| .
$$

Let us make use of the definition of the numerical scheme (3.2), (3.3). Applying the mean value theorem to the function $f$ we obtain

$$
\begin{aligned}
\rho_{j}^{n+1}-\rho_{j}^{n}= & \frac{\lambda \alpha}{2}\left(\rho_{j-1}^{n}-\rho_{j}^{n}\right)+\frac{\lambda \alpha}{2}\left(\rho_{j+1}^{n}-\rho_{j}^{n}\right)+\frac{\lambda}{2}\left(f\left(\rho_{j-1}^{n}\right) V_{j-1}^{n} \pm f\left(\rho_{j-1}^{n}\right) V_{j+1}^{n}-V_{j+1}^{n} f\left(\rho_{j+1}^{n}\right)\right) \\
= & \frac{\lambda}{2}\left(\alpha+V_{j+1}^{n} f^{\prime}\left(\zeta_{j-1 / 2}\right)\right)\left(\rho_{j-1}^{n}-\rho_{j}^{n}\right) \\
& +\frac{\lambda}{2}\left(-\alpha+V_{j+1}^{n} f^{\prime}\left(\zeta_{j+1 / 2}\right)\right)\left(\rho_{j}^{n}-\rho_{j+1}^{n}\right) \\
& +\frac{\lambda}{2} f\left(\rho_{j-1}^{n}\right)\left(V_{j-1}^{n}-V_{j}^{n}\right)+\frac{\lambda}{2} f\left(\rho_{j-1}^{n}\right)\left(V_{j}^{n}-V_{j+1}^{n}\right),
\end{aligned}
$$

where $\zeta_{j-1 / 2}$ is between $\rho_{j-1}^{n}$ and $\rho_{j}^{n}$. Applying again the mean value theorem, we obtain

$$
V_{j-1}^{n}-V_{j}^{n}=v^{\prime}\left(\xi_{j-1 / 2}\right) \Delta x \sum_{k=0}^{N-1} J_{\gamma}^{k}\left(\rho_{j+k-1}^{n}-\rho_{j+k}^{n}\right)
$$


and

$$
V_{j}^{n}-V_{j+1}^{n}=v^{\prime}\left(\xi_{j+1 / 2}\right) \Delta x \sum_{k=0}^{N-1} J_{\gamma}^{k}\left(\rho_{j+k}^{n}-\rho_{j+k+1}^{n}\right) .
$$

Therefore we can write

$$
\begin{aligned}
\rho_{j}^{n+1}-\rho_{j}^{n}= & \frac{\lambda}{2}\left(\alpha+V_{j+1}^{n} f^{\prime}\left(\zeta_{j-1 / 2}\right)+f\left(\rho_{j-1}^{n}\right) v^{\prime}\left(\xi_{j-1 / 2}\right) \Delta x J_{\gamma}^{0}\right)\left(\rho_{j-1}^{n}-\rho_{j}^{n}\right) \\
& +\frac{\lambda}{2}\left(-\alpha+V_{j+1}^{n} f^{\prime}\left(\zeta_{j+1 / 2}\right)+f\left(\rho_{j-1}^{n}\right) v^{\prime}\left(\xi_{j-1 / 2}\right) \Delta x J_{\gamma}^{1}+f\left(\rho_{j-1}^{n}\right) v^{\prime}\left(\xi_{j+1 / 2}\right) \Delta x J_{\gamma}^{0}\right)\left(\rho_{j}^{n}-\rho_{j+1}^{n}\right) \\
& +\frac{\lambda}{2} f\left(\rho_{j-1}^{n}\right) v^{\prime}\left(\xi_{j-1 / 2}\right) \Delta x \sum_{k=2}^{N-1} J_{\gamma}^{k}\left(\rho_{j+k-1}^{n}-\rho_{j+k}^{n}\right) \\
& +\frac{\lambda}{2} f\left(\rho_{j-1}^{n}\right) v^{\prime}\left(\xi_{j+1 / 2}\right) \Delta x \sum_{k=1}^{N-1} J_{\gamma}^{k}\left(\rho_{j+k}^{n}-\rho_{j+k+1}^{n}\right) .
\end{aligned}
$$

Rearranging the indexes of the last two terms, we can write

$$
\begin{aligned}
\rho_{j}^{n+1}-\rho_{j}^{n}= & \frac{\lambda}{2}\left(\alpha+V_{j+1}^{n} f^{\prime}\left(\zeta_{j-1 / 2}\right)+f\left(\rho_{j-1}^{n}\right) v^{\prime}\left(\xi_{j-1 / 2}\right) \Delta x J_{\gamma}^{0}\right)\left(\rho_{j-1}^{n}-\rho_{j}^{n}\right) \\
& -\frac{\lambda}{2}\left(\alpha-V_{j+1}^{n} f^{\prime}\left(\zeta_{j+1 / 2}\right)-f\left(\rho_{j-1}^{n}\right) v^{\prime}\left(\xi_{j-1 / 2}\right) \Delta x J_{\gamma}^{1}-f\left(\rho_{j-1}^{n}\right) v^{\prime}\left(\xi_{j+1 / 2}\right) \Delta x J_{\gamma}^{0}\right)\left(\rho_{j}^{n}-\rho_{j+1}^{n}\right) \\
& +\frac{\lambda}{2} f\left(\rho_{j-1}^{n}\right) \Delta x \sum_{k=1}^{N-2}\left(v^{\prime}\left(\xi_{j-1 / 2}\right) J_{\gamma}^{k+1}+v^{\prime}\left(\xi_{j+1 / 2}\right) J_{\gamma}^{k}\right)\left(\rho_{j+k}^{n}-\rho_{j+k+1}^{n}\right) \\
& +\frac{\lambda}{2} f\left(\rho_{j-1}^{n}\right) v^{\prime}\left(\xi_{j+1 / 2}\right) \Delta x J_{\gamma}^{N-1}\left(\rho_{j+N-1}^{n}-\rho_{j+N}^{n}\right) .
\end{aligned}
$$

Observe that the coefficients in (3.20a) and (3.20b) are positive if $\alpha \geq\left\|f^{\prime}\right\|\|v\|+\Delta x J_{\gamma}(0)\|f\|\left\|v^{\prime}\right\|$. Therefore, taking the absolute values in (3.20) we get

$$
\begin{aligned}
\left|\rho_{j}^{n+1}-\rho_{j}^{n}\right|= & \frac{\lambda}{2}\left(\alpha+V_{j+1}^{n} f^{\prime}\left(\zeta_{j-1 / 2}\right)+f\left(\rho_{j-1}^{n}\right) v^{\prime}\left(\xi_{j-1 / 2}\right) \Delta x J_{\gamma}^{0}\right)\left|\rho_{j-1}^{n}-\rho_{j}^{n}\right| \\
& +\frac{\lambda}{2}\left(\alpha-V_{j+1}^{n} f^{\prime}\left(\zeta_{j+1 / 2}\right)-f\left(\rho_{j-1}^{n}\right) v^{\prime}\left(\xi_{j-1 / 2}\right) \Delta x J_{\gamma}^{1}-f\left(\rho_{j-1}^{n}\right) v^{\prime}\left(\xi_{j+1 / 2}\right) \Delta x J_{\gamma}^{0}\right)\left|\rho_{j}^{n}-\rho_{j+1}^{n}\right| \\
& -\frac{\lambda}{2} f\left(\rho_{j-1}^{n}\right) \Delta x \sum_{k=1}^{N-2}\left(v^{\prime}\left(\xi_{j-1 / 2}\right) J_{\gamma}^{k+1}+v^{\prime}\left(\xi_{j+1 / 2}\right) J_{\gamma}^{k}\right)\left|\rho_{j+k}^{n}-\rho_{j+k+1}^{n}\right| \\
& -\frac{\lambda}{2} f\left(\rho_{j-1}^{n}\right) v^{\prime}\left(\xi_{j+1 / 2}\right) \Delta x J_{\gamma}^{N-1}\left|\rho_{j+N-1}^{n}-\rho_{j+N}^{n}\right| .
\end{aligned}
$$

Summing on $j$ and rearranging the indexes we obtain

$$
\begin{aligned}
\sum_{j \in \mathbb{Z}} \Delta x\left|\rho_{j}^{n+1}-\rho_{j}^{n}\right| \leq & \frac{\Delta t}{2} \sum_{j \in \mathbb{Z}}\left|\rho_{j+1}^{n}-\rho_{j}^{n}\right| \\
& \times\left[2 \alpha+f^{\prime}\left(\zeta_{j+1 / 2}\right)\left(V_{j+2}^{n}-V_{j+1}^{n}\right)\right. \\
& +\Delta x f\left(\rho_{j}^{n}\right) v^{\prime}\left(\xi_{j+1 / 2}\right) J_{\gamma}^{0}-\Delta x f\left(\rho_{j-1}^{n}\right) v^{\prime}\left(\xi_{j-1 / 2}\right) J_{\gamma}^{1}
\end{aligned}
$$




$$
\begin{aligned}
& -\Delta x f\left(\rho_{j-1}^{n}\right) v^{\prime}\left(\xi_{j+1 / 2}\right) J_{\gamma}^{0}-\Delta x \sum_{k=1}^{N-2} f\left(\rho_{j-k-1}^{n}\right)\left(v^{\prime}\left(\xi_{j-k-1 / 2}\right) J_{\gamma}^{k+1}+v^{\prime}\left(\xi_{j-k+1 / 2}\right) J_{\gamma}^{k}\right) \\
& \left.-\Delta x f\left(\rho_{j-N}^{n}\right) v^{\prime}\left(\xi_{j-N+3 / 2}\right) J_{\gamma}^{N-1}\right] \\
& \leq \frac{\Delta t}{2} \sum_{j \in \mathbb{Z}}\left|\rho_{j+1}^{n}-\rho_{j}^{n}\right|\left(2 \alpha+\Delta x J_{\gamma}(0)\left\|v^{\prime}\right\|\left(3\|f\|+\left\|f^{\prime}\right\|\left\|\rho_{0}\right\|\right)+2 J_{0}\|f\|\left\|v^{\prime}\right\|\right)
\end{aligned}
$$

which yields

$$
\sum_{n=0}^{M-1} \sum_{j \in \mathbb{Z}} \Delta x\left|\rho_{j}^{n+1}-\rho_{j}^{n}\right| \leq T e^{C\left(J_{\gamma}\right) T}\left(\alpha+\frac{1}{2} \Delta x J_{\gamma}(0)\left\|v^{\prime}\right\|\left(3\|f\|+\left\|f^{\prime}\right\|\left\|\rho_{0}\right\|\right)+J_{0}\|f\|\left\|v^{\prime}\right\|\right) \operatorname{TV}\left(\rho_{0}\right),
$$

since $M \Delta t<T$. Taking $\alpha=\left\|f^{\prime}\right\|\|v\|+\Delta x J_{\gamma}(0)\|f\|\left\|v^{\prime}\right\|$, we obtain the bound (3.18) with

$$
\tilde{C}=T e^{C\left(J_{\gamma}\right) T}\left(1+\left\|f^{\prime}\right\|\|v\|+\frac{1}{2} \Delta x J_{\gamma}(0)\left\|v^{\prime}\right\|\left(5\|f\|+\left\|f^{\prime}\right\|\left\|\rho_{0}\right\|\right)+J_{0}\|f\|\left\|v^{\prime}\right\|\right) \operatorname{TV}\left(\rho_{0}\right) .
$$

Note that (3.21) allows to recover (1.6b) as $\Delta x \rightarrow 0$.

\subsection{Discrete entropy inequalities}

Following $[2,4,10]$, we derive a discrete entropy inequality for the approximate solution generated by (3.2), (3.3), which is used to prove that the limit of Lax-Friedrichs approximations is indeed a weak entropy solution in the sense of Definition 1 . We denote

$$
\begin{gathered}
G_{j+1 / 2}(u, w):=\frac{1}{2} f(u) V_{j}^{n}+\frac{1}{2} f(w) V_{j+1}^{n}+\frac{\alpha}{2}(u-w), \\
F_{j+1 / 2}^{\kappa}(u, w):=G_{j+1 / 2}(u \wedge \kappa, w \wedge \kappa)-G_{j+1 / 2}(u \vee \kappa, w \vee \kappa),
\end{gathered}
$$

with $a \wedge b=\max (a, b)$ and $a \vee b=\min (a, b)$.

Proposition 3. Under hypotheses $(\mathbf{H})$, let $\rho_{j}^{n}, j \in \mathbb{Z}, n \in \mathbb{N}$, be given by (3.2), (3.3). Then, if $\alpha \geq\left\|f^{\prime}\right\|\|v\|$ and $\lambda \leq 1 / \alpha$, we have

$$
\begin{aligned}
\left|\rho^{n+1_{j}}-\kappa\right|-\left|\rho_{j}^{n}-\kappa\right|+\lambda( & \left.F_{j+1 / 2}^{\kappa}\left(\rho_{j}^{n}, \rho_{j+1}^{n}\right)-F_{j-1 / 2}^{\kappa}\left(\rho_{j-1}^{n}, \rho_{j}^{n}\right)\right) \\
& +\frac{\lambda}{2} \operatorname{sgn}\left(\rho_{j}^{n+1}-k\right) f(\kappa)\left(V_{j+1}^{n}-V_{j-1}^{n}\right) \leq 0,
\end{aligned}
$$

for all $j \in \mathbb{Z}, n \in \mathbb{N}$, and $\kappa \in \mathbb{R}$.

Proof. The proof follows closely $[2,4]$. We detail it below for sake of completeness. We set

$$
\tilde{H}_{j}(u, w, z)=w-\lambda\left(G_{j+1 / 2}(w, z)-G_{j-1 / 2}(u, w)\right) .
$$

The function $\tilde{H}_{j}$ is monotone non-decreasing with respect to each variable for $\alpha \lambda \leq 1$ and $\alpha \geq$ $\left\|f^{\prime}\right\|\|v\|$, which are guaranteed by (3.5) and (3.6). Indeed, we have

$$
\tilde{H}_{j}(u, w, z)=w-\frac{\lambda}{2}\left(f(z) V_{j+1}^{n}-f(u) V_{j-1}^{n}+\alpha(2 w-u-z)\right),
$$


so the partial derivatives are

$$
\begin{aligned}
\frac{\partial \tilde{H}_{j}}{\partial u} & =\frac{\lambda}{2}\left(f^{\prime}(u) V_{j-1}^{n}+\alpha\right), \\
\frac{\partial \tilde{H}_{j}}{\partial w} & =1-\lambda \alpha, \\
\frac{\partial \tilde{H}_{j}}{\partial z} & =\frac{\lambda}{2}\left(\alpha-f^{\prime}(z) V_{j+1}^{n}\right) .
\end{aligned}
$$

Moreover, we have the identity

$$
\begin{gathered}
\tilde{H}_{j}\left(\rho_{j-1}^{n} \wedge \kappa, \rho_{j}^{n} \wedge \kappa, \rho_{j+1}^{n} \wedge \kappa\right)-\tilde{H}_{j}\left(\rho_{j-1}^{n} \vee \kappa, \rho_{j}^{n} \vee \kappa, \rho_{j+1}^{n} \vee \kappa\right) \\
=\left|\rho_{j}^{n}-\kappa\right|-\lambda\left(F_{j+1 / 2}^{\kappa}\left(\rho_{j}^{n}, \rho_{j+1}^{n}\right)-F_{j-1 / 2}^{\kappa}\left(\rho_{j-1}^{n}, \rho_{j}^{n}\right)\right) .
\end{gathered}
$$

By monotonicity,

$$
\begin{aligned}
& \tilde{H}_{j}\left(\rho_{j-1}^{n} \wedge \kappa, \rho_{j}^{n} \wedge \kappa, \rho_{j+1}^{n} \wedge \kappa\right)-\tilde{H}_{j}\left(\rho_{j-1}^{n} \vee \kappa, \rho_{j}^{n} \vee \kappa, \rho_{j+1}^{n} \vee \kappa\right) \\
\geq & \tilde{H}_{j}\left(\rho_{j-1}^{n}, \rho_{j}^{n}, \rho_{j+1}^{n}\right) \wedge \tilde{H}_{j}(\kappa, \kappa, \kappa)-\tilde{H}_{j}\left(\rho_{j-1}^{n}, \rho_{j}^{n}, \rho_{j+1}^{n}\right) \vee \tilde{H}_{j}(\kappa, \kappa, \kappa) \\
= & \left|\tilde{H}_{j}\left(\rho_{j-1}^{n}, \rho_{j}^{n}, \rho_{j+1}^{n}\right)-\tilde{H}_{j}(\kappa, \kappa, \kappa)\right| \\
= & \operatorname{sgn}\left(\tilde{H}_{j}\left(\rho_{j-1}^{n}, \rho_{j}^{n}, \rho_{j+1}^{n}\right)-\tilde{H}_{j}(\kappa, \kappa, \kappa)\right) \times\left(\tilde{H}_{j}\left(\rho_{j-1}^{n}, \rho_{j}^{n}, \rho_{j+1}^{n}\right)-\tilde{H}_{j}(\kappa, \kappa, \kappa)\right) \\
= & \operatorname{sgn}\left(\tilde{H}_{j}\left(\rho_{j-1}^{n}, \rho_{j}^{n}, \rho_{j+1}^{n}\right)-\kappa+\frac{\lambda}{2} f(\kappa)\left(V_{j+1}^{n}-V_{j-1}^{n}\right)\right) \\
& \times\left(\tilde{H}_{j}\left(\rho_{j-1}^{n}, \rho_{j}^{n}, \rho_{j+1}^{n}\right)-\kappa+\frac{\lambda}{2} f(\kappa)\left(V_{j+1}^{n}-V_{j-1}^{n}\right)\right) \\
\geq & \operatorname{sgn}\left(\tilde{H}_{j}\left(\rho_{j-1}^{n}, \rho_{j}^{n}, \rho_{j+1}^{n}\right)-\kappa\right) \times\left(\tilde{H}_{j}\left(\rho_{j-1}^{n}, \rho_{j}^{n}, \rho_{j+1}^{n}\right)-\kappa+\frac{\lambda}{2} f(\kappa)\left(V_{j+1}^{n}-V_{j-1}^{n}\right)\right) \\
= & \left|\tilde{H}_{j}\left(\rho_{j-1}^{n}, \rho_{j}^{n}, \rho_{j+1}^{n}\right)-\kappa\right|+\frac{\lambda}{2} \operatorname{sgn}\left(\tilde{H}_{j}\left(\rho_{j-1}^{n}, \rho_{j}^{n}, \rho_{j+1}^{n}\right)-\kappa\right) f(\kappa)\left(V_{j+1}^{n}-V_{j-1}^{n}\right) \\
= & \left|\rho_{j}^{n+1}-\kappa\right|+\frac{\lambda}{2} \operatorname{sgn}\left(\rho_{j}^{n+1}-\kappa\right) f(\kappa)\left(V_{j+1}^{n}-V_{j-1}^{n}\right),
\end{aligned}
$$

by definition of the scheme (3.2), (3.3), which gives (3.22).

Proof of Theorem 1. Thanks to Proposition 1 and Corollary 4, we can apply Helly's theorem stating that there exists a subsequence $\rho_{\Delta x}$ that converges to some $\rho \in\left(L^{1} \cap L^{\infty} \cap \mathrm{BV}\right)\left(\mathbb{R}^{+} \times \mathbb{R} ; I\right)$ in the $\mathbf{L}_{\text {loc }}^{1}$-norm. One can then follow a Lax-Wendroff type argument to show that the limit function $\rho$ is a weak entropy solution of (1.1), (1.3), in the sense of Definition 1. We just observe that the numerical flux also depends on $\Delta x$, therefore the classical argument on flux consistency and Lipschitz dependence must be replaced by direct estimates, like in $[4,9]$.

Proof of Corollary 2. When the look-ahead distance $\gamma \rightarrow \infty$, the non-local flux in (1.1) becomes a local one. Since the bounds (1.5), (1.6) are uniform as $\gamma \rightarrow \infty$, the solution $\rho^{\gamma}$ of problem (1.1), (3.3), tends up to a subsequence to the solution $\rho$ of the local problem (1.7) in the $\mathbf{L}_{\text {loc }}^{1}$-norm when $\gamma \rightarrow \infty$. In fact, applying Lebesgue's dominated convergence theorem in (1.4), since

$$
\left|\operatorname{sgn}(\rho-\kappa)(f(\rho)-f(\kappa)) v\left(J_{\gamma} * \rho\right)\right| \leq 2|f|\|v\|
$$


and

$$
\left|\operatorname{sgn}(\rho-\kappa) f(\kappa) v^{\prime}\left(J_{\gamma} * \rho\right) \partial_{x}\left(J_{\gamma} * \rho\right)\right| \leq 3|f|\|\rho\|\left\|J_{\gamma}\right\|\left\|v^{\prime}\right\|
$$

we obtain

$$
\int_{0}^{+\infty} \int_{\mathbb{R}}\left\{|\rho-\kappa| \varphi_{t}+\operatorname{sgn}(\rho-\kappa)(f(\rho)-f(\kappa)) v(0) \varphi_{x}+\int_{\mathbb{R}}\left|\rho_{0}(x)-\kappa\right| \varphi(0, x) d x \geq 0,\right.
$$

which is the definition of entropy weak solution for the classical equation (1.7).

\section{Numerical tests}

In this section, we perform some numerical simulations to illustrate the result of Corollary 2, taking two different choices for the speed law $v$, the convolution kernel $J_{\gamma}$ and the function $f$. More precisely, we consider the models studied in [15, 18] and [4], which consist in the following equations:

$$
\partial_{t} \rho+\partial_{x}\left(\rho(1-\rho) e^{-\left(J_{\gamma} * \rho\right)}\right)=0, \quad x \in \mathbb{R}, t>0,
$$

for the Arrhenius look-ahead dynamics [18], and

$$
\partial_{t} \rho+\partial_{x}\left(\rho\left(1-J_{\gamma} * \rho\right)\right)=0, \quad x \in \mathbb{R}, t>0,
$$

for the Lighthill-Whitham-Richards (LWR) model with non-local velocity [4].

Equations (4.1) and (4.2) correspond to the following choices of $f \in \mathbf{C}^{\mathbf{1}}\left([0,1] ; \mathbb{R}^{+}\right)$and $v \in$ $\mathbf{C}^{2}\left([0,1] ; \mathbb{R}^{+}\right)$:

$$
\begin{array}{ll}
f(\rho)=\rho(1-\rho), & v(\rho)=e^{-\rho}, \\
f(\rho)=\rho, & v(\rho)=(1-\rho),
\end{array}
$$

respectively. Besides, we will consider the following kernels $J_{\gamma} \in \mathbf{C}^{\mathbf{1}}\left([0, \gamma] ; \mathbb{R}^{+}\right)$, see $[4,14]$ :

$$
\begin{array}{lll}
\text { constant: } & J_{\gamma}(x)=\frac{1}{\gamma}, \\
\text { linear decreasing: } & J_{\gamma}(x)=\frac{2}{\gamma}\left(1-\frac{x}{\gamma}\right) .
\end{array}
$$

For the tests, the space domain is given by the interval $[-1,1]$ and the space discretization mesh is $\Delta x=0.001$. We impose absorbing conditions at the boundaries, adding $N=\gamma / \Delta x$ ghost cells at the right boundary and just one at the left, where we extend the solution constantly equal to the last value inside the domain. Our aim is to investigate the convergence of (4.3) to the solution of the LWR model $[16,17]$

$$
\partial_{t} \rho+\partial_{x}(\rho(1-\rho))=0
$$

and the convergence of (4.4) to the solution of the transport equation

$$
\partial_{t} \rho+\partial_{x} \rho=0
$$

as $\gamma \rightarrow \infty$. We study both problems with the initial datum

$$
\rho_{0}(x)= \begin{cases}0.8 & \text { for }-0.5<x<-0.1, \\ 0 & \text { otherwise }\end{cases}
$$

that describes the case of a red traffic light located at $x=-0.1$, which turns green at the initial time $t=0$. Figures 1 and 2 illustrate the behavior for models (4.1) and (4.2), respectively, in agreement with the theoretical results. 


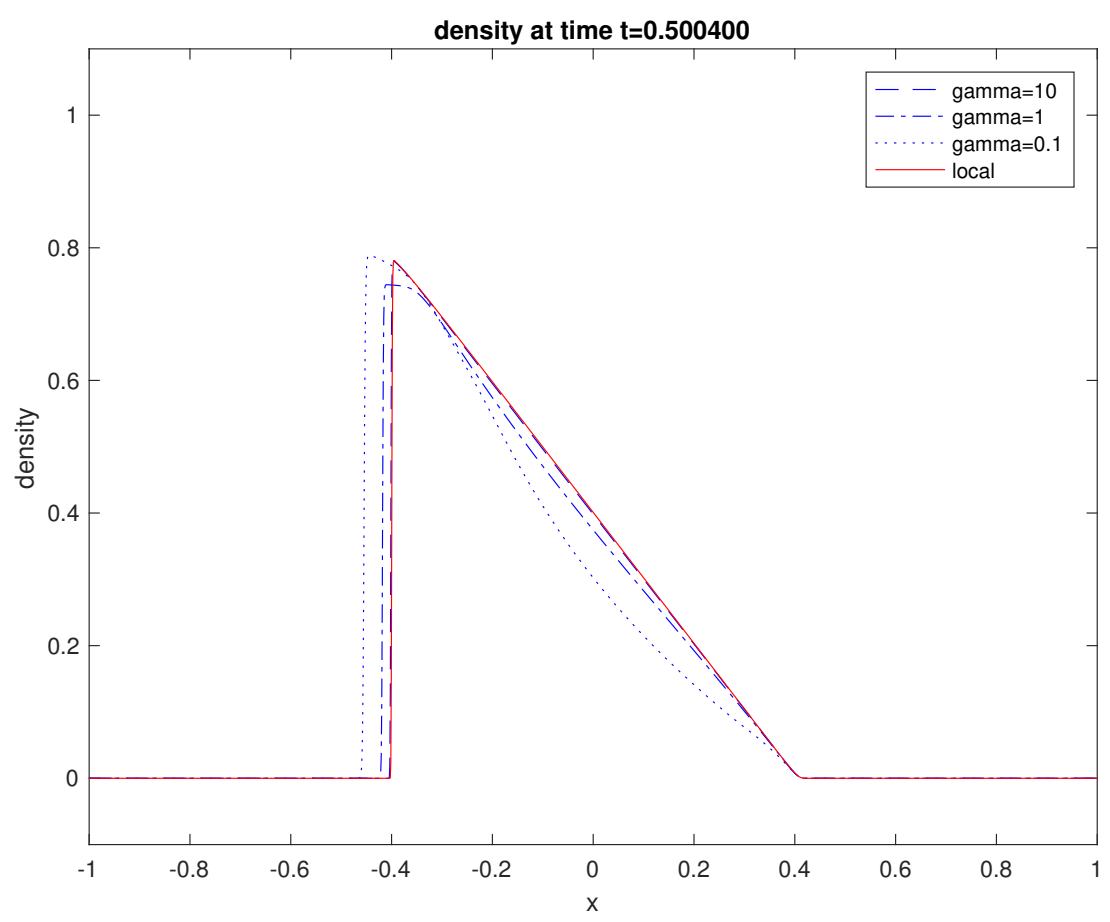

(a) $J_{\gamma}$ constant

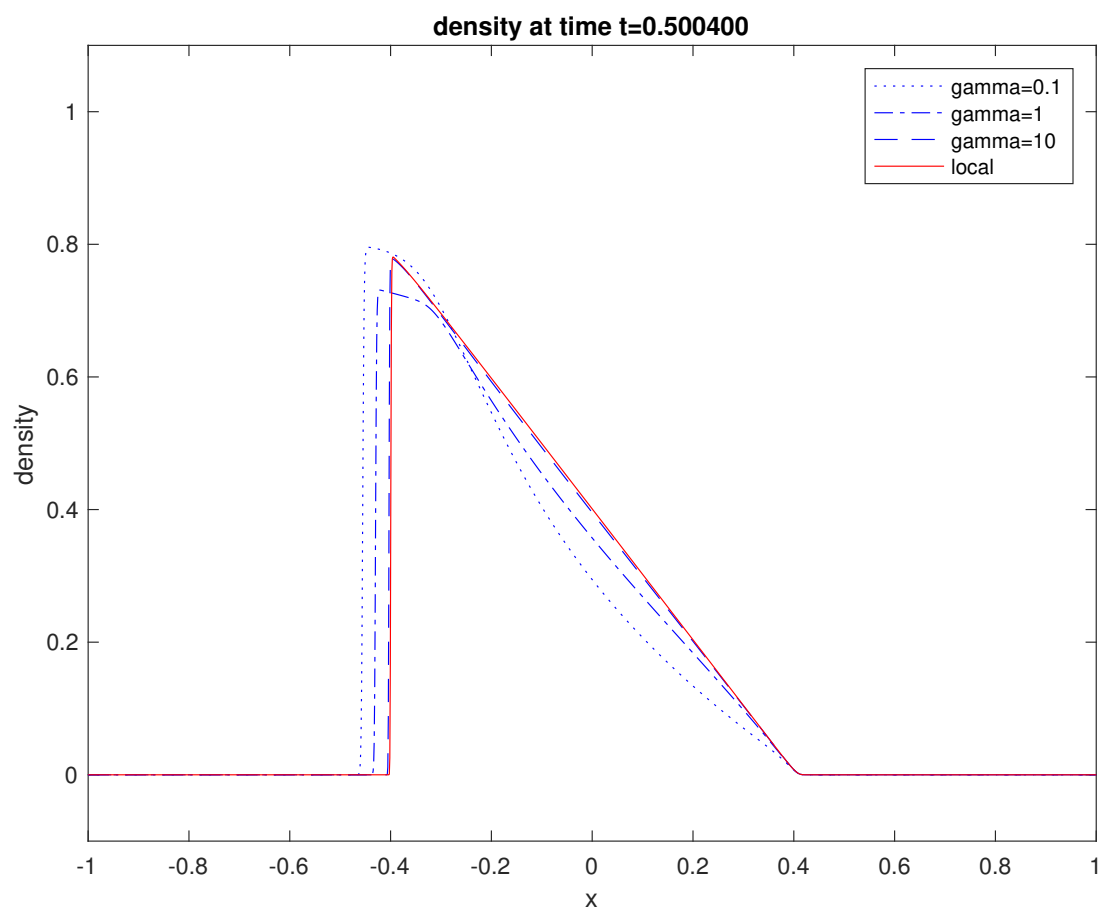

(b) $J_{\gamma}$ linear decreasing

Figure 1: Density profiles corresponding to the non-local equation (4.1) with increasing values of $\gamma=0.1,1,10$. We can observe that the nonlocal solution tends to the solution of (4.5) (red line) as $\gamma \rightarrow \infty$. 


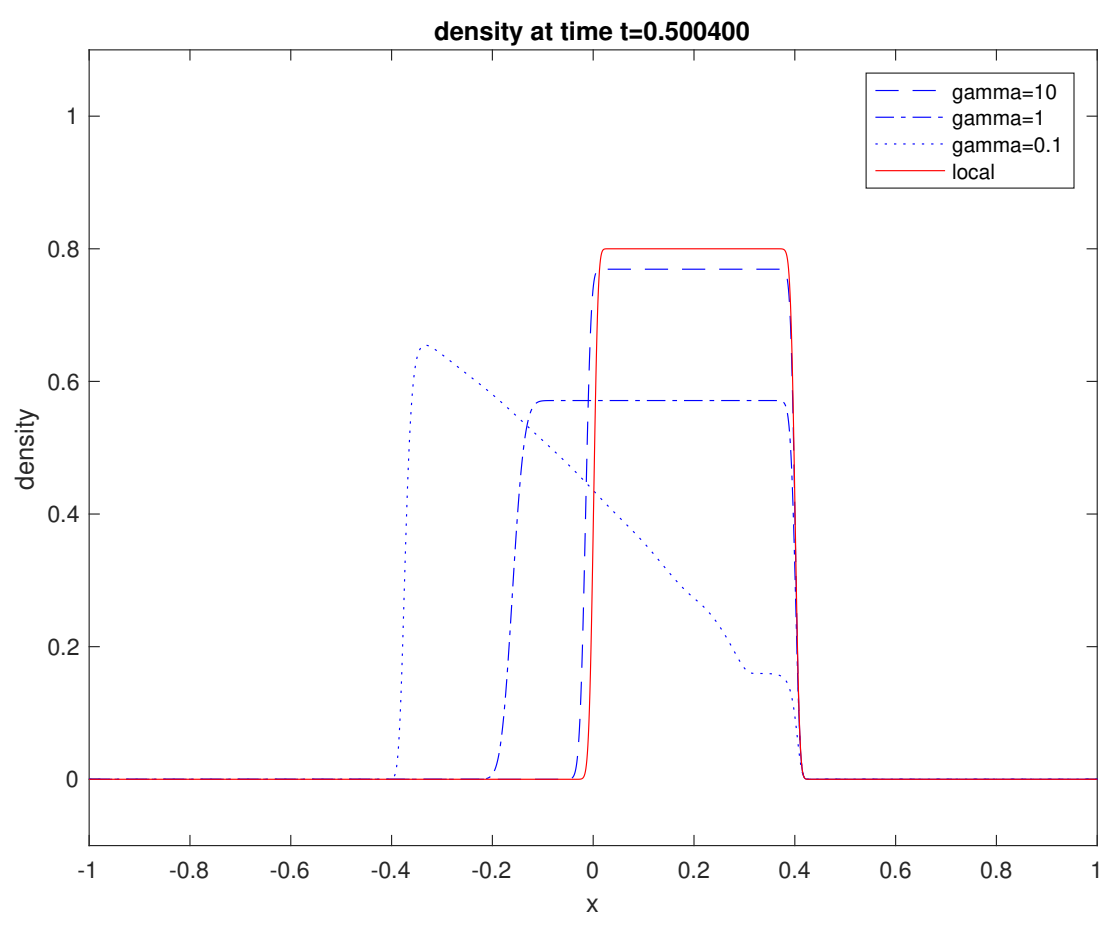

(a) $J_{\gamma}$ constant

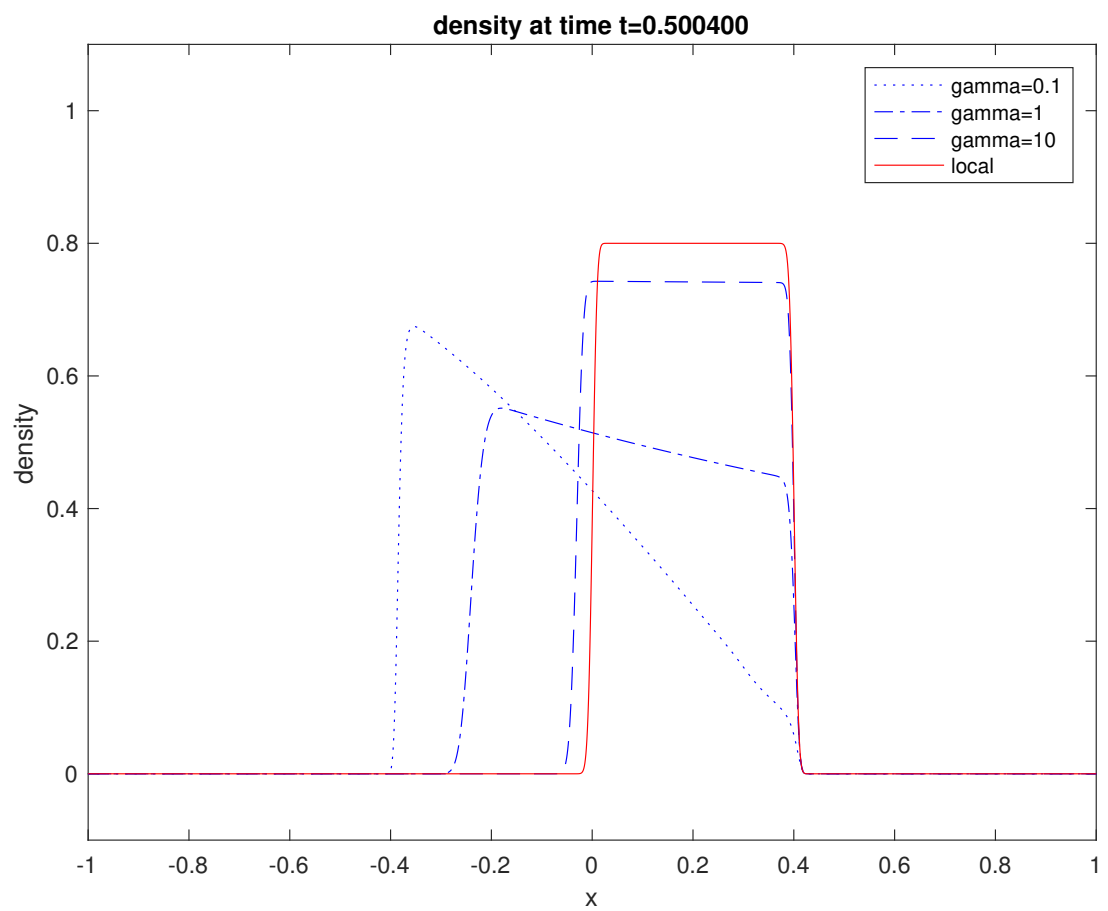

(b) $J_{\gamma}$ linear decreasing

Figure 2: Density profiles corresponding to the non-local equation (4.2) with increasing values of $\gamma=0.1,1,10$. We can observe that the nonlocal solution tends to the solution of (4.6) (red line) as $\gamma \rightarrow \infty$. 


\section{References}

[1] A. Aggarwal, R. M. Colombo, and P. Goatin. Nonlocal systems of conservation laws in several space dimensions. SIAM Journal on Numerical Analysis, 53(2):963-983, 2015.

[2] P. Amorim, R. M. Colombo, and A. Teixeira. On the numerical integration of scalar nonlocal conservation laws. ESAIM M2AN, 49(1):19-37, 2015.

[3] F. Betancourt, R. Bürger, K. H. Karlsen, and E. M. Tory. On nonlocal conservation laws modelling sedimentation. Nonlinearity, 24(3):855-885, 2011.

[4] S. Blandin and P. Goatin. Well-posedness of a conservation law with non-local flux arising in traffic flow modeling. Numer. Math., 132(2):217-241, 2016.

[5] R. M. Colombo, M. Garavello, and M. Lécureux-Mercier. A class of nonlocal models for pedestrian traffic. Mathematical Models and Methods in Applied Sciences, 22(04):1150023, 2012.

[6] R. M. Colombo and F. Marcellini. Nonlocal systems of balance laws in several space dimensions with applications to laser technology. J. Differential Equations, 259(11):6749-6773, 2015.

[7] R. M. Colombo and E. Rossi. Hyperbolic predators vs. parabolic prey. Commun. Math. Sci., 13(2):369400, 2015.

[8] R. Eymard, T. Gallouët, and R. Herbin. Finite volume methods. In Handbook of numerical analysis, Vol. VII, Handb. Numer. Anal., VII, pages 713-1020. North-Holland, Amsterdam, 2000.

[9] P. Goatin and S. Scialanga. The Lighthill-Whitham-Richards traffic flow model with non-local velocity: analytical study and numerical results. Research Report RR-8685, Inria Sophia Antipolis, Feb. 2015.

[10] P. Goatin and S. Scialanga. Well-posedness and finite volume approximations of the LWR traffic flow model with non-local velocity. Netw. Heterog. Media, 11(1):107-121, 2016.

[11] S. Göttlich, S. Hoher, P. Schindler, V. Schleper, and A. Verl. Modeling, simulation and validation of material flow on conveyor belts. Applied Mathematical Modelling, 38(13):3295 - 3313, 2014.

[12] A. Keimer and L. Pflug. Existence, uniqueness and regularity results on nonlocal balance laws. $J$. Differential Equations, 263(7):4023-4069, 2017.

[13] S. N. Kružkov. First order quasilinear equations with several independent variables. Mat. Sb. (N.S.), 81 (123):228-255, 1970.

[14] A. Kurganov and A. Polizzi. Non-oscillatory central schemes for a traffic flow model with Arrehenius look-ahead dynamics. Netw. Heterog. Media, 4(3):431-451, 2009.

[15] D. Li and T. Li. Shock formation in a traffic flow model with Arrhenius look-ahead dynamics. Networks and Heterogeneous Media, 6(4):681-694, 2011.

[16] M. J. Lighthill and G. B. Whitham. On kinematic waves. II. A theory of traffic flow on long crowded roads. Proc. Roy. Soc. London. Ser. A., 229:317-345, 1955.

[17] P. I. Richards. Shock waves on the highway. Operations Res., 4:42-51, 1956.

[18] A. Sopasakis and M. A. Katsoulakis. Stochastic modeling and simulation of traffic flow: asymmetric single exclusion process with Arrhenius look-ahead dynamics. SIAM J. Appl. Math., 66(3):921-944 (electronic), 2006.

[19] K. Zumbrun. On a nonlocal dispersive equation modeling particle suspensions. Quart. Appl. Math., $57(3): 573-600,1999$. 